\title{
Remoción de cromo total en agua, usando carbón mineral activado con radiación de microondas
}

Resumen: Se estudió la remoción del cromo para validar el uso del carbón mineral activado mediante radiación de microondas, dicha técnica de activación fue usada anteriormente en la oxidación de nanotubos de carbono y carbón vegetal. Se buscó aplicar esta técnica en un carbón de origen mineral, procedente de las minas de carbón de la cuenca del Rio Santa - Ancash y mejorar las propiedades de remoción de dicho material, con lo que a largo plazo y a mayor investigación se lograría darle un uso aparte de la que actualmente es destinada. Las pruebas experimentales de activación resultaron exitosas, esto, reflejados en las cinéticas de remoción del cromo; los valores de capacidad de remoción determinadas en dichas cinéticas están entre el $34 \%$ para el carbón sin activar y el $41 \%$ para el carbón activado, la que indica que la capacidad de remoción depende mucho de la activación del material.

Palabras-claves: Carbón mineral; activación de carbón por microondas; Cinética.

REMOVAL TOTAL CHROMIUM IN WATER USING ACTIVATED COAL MICROWAVE RADIATION

Abstract: Removal of chromium was studied to validate the use of activated carbon by microwave radiation. This activation technique was previously used in the oxidation of carbon nanotubes and charcoal. It was tried to apply this technique in a coal of mineral origin, coming from the coal mines of the Santa - Ancash river basin and to improve the properties of removal of this material, with which in the long term and to greater investigation would be possible to give a use Apart from the one that is currently destined. The experimental activation tests were successful, this reflected in the removal kinetics of chromium; the removal capacity values determined in said kinetics are between $34 \%$ for unconverted coal and $41 \%$ for activated charcoal, which indicates that the removal capacity is highly dependent on the activation of the material.

Keywords: Mineral coal; activated coal in microwave; Kinetic.

\section{INTRODUCCIÓN}

El cromo es un elemento de origen natural el cual existe en 9 estados de valencia con un rango de $+2 \mathrm{a}+6$, las más comunes son en estados de cromo (0) estado metálico, la que se usa en la fabricación del acero, cromo (VI) y (III) los que se usan en cromado, biocida en sistemas de enfriamiento de aguas, explosivos, colorantes y pigmentos, curtido de cueros y preservación de madera. (ATSDR, 2008)

Los únicos compuestos de importancia biológica son los derivados de los estados de oxidación +3 y +6 ; al primer grupo pertenecen el óxido crómico () y las sales crómicas como el cloruro crómico ) o el anión cromito () y, al segundo grupo, el trióxido de cromo () los cromatos y los dicromatos. (Palacios y América, 1997)

El carbón, un material usado desde la antigüedad, se conoce que desde el año 1550 a.C. se usaba dicho material como adsorbente para determinadas prácticas médicas, así mismo en el año 400 a.C. Hipócrates recomendaba filtrar el agua con carbón vegetal para eliminar malos olores y sabores y para prevenir enfermedades. Ya en el año 450 a.C. se trataba el agua con carbón activado. (Universidad de Sevilla, 2009)

La literatura ofrece un sin fin de propiedades y usos que se le da al carbón activado. Es asertivo indicar que el carbón activado es un material adsorbente por excelencia, por sus usos en la remoción de contaminantes en al agua, aire, tratamiento de intoxicaciones en personas, controlador de olores, etc.

Cabe decir que dicho material puede tener origen vegetal y mineral y una de las características para poder activarlas, es el de ser un material suave llamado en proceso de coquización, esto responde al orden de sus moléculas; mientras más desordenados sean sus átomos, el carbón es menos duro y más fácil de poder activarse. (Carbotecnia, 2016)

El carbón puede activarse mediante procesos térmicos o químicos, estos provocando oxidación parcial del carbón que ayuden a formar poros y rompiendo uniones que unen la celulosa usando ácidos; esta última técnica usada en el carbón vegetal.

Ingeniero Mecánico de Fluidos UNMSM, jdelatorre@unmsm.edu.pe, Docente UNMSM

Ingeniero Ambiental, egresado Maestría Ciencias Ambientales UNMSM, dechevarria@unmsm. edu.pe Director Asuntos Ambientales DREM Amazonas.

Química, UNMSM, jalvarez@unmsm.edu.pe, Docente UNMSM

4 Ingeniera Química UNMSM, nancy.barreda@unmsm.edu.pe, Docente UNMSM 
El Perú cuenta con buenas reservas de carbón mineral, distribuidos en cuencas, como son: Alto Chicama en La Libertad, El Santa en Ancash, Jatunhuasi en Junín, Piñipata en Cajamarca, Oyón en Lima, Yanacancha en Cajamarca, etc. (Aramburú, Núñez, Azañero, León, Fernández, Gagliuffi, 2007)

Estas cuencas sirven de referencia para poder identificar el tipo de carbón que se está usando en la presente investigación, el material usado tiene como origen la cuenca del Rio Santa (Distrito de Huallanca - Provincia de Huaylas, departamento de Ancash), las características generales de este tipo de carbón le dan una calificación de carbón Antracita - Metantracita. (Agramonte y Díaz, 1983)

El uso de radiación de microondas en la activación del carbón, ayuda a suministrar energía directamente al esqueleto del carbono a nivel molecular, reduciendo las etapas habituales de activación; por lo que el método es una fuente que proporciona ventajas como el ahorro de energía y reducción de tiempo de procesamiento, calentamiento selectivo y la mejora de la estructura de los poros del material. (Xuemin, Dandan, Yujun, Zhilin, Zhansheng, y Giancarlo, 2015)

El calentamiento por microondas, es una técnica atractiva que realiza el calentamiento interno y volumétrico del material; las microondas tienen la capacidad de acelerar la velocidad de las reacciones químicas, generando gases como ,, y, las que aumentan los poros del carbón, al tiempo que reduce el consumo de energía para generar el calentamiento, comparándola con una activación convencional por calor. (Vaishakh y Vinu, 2016)

Así mismo el uso del peróxido de hidrógeno al 30\% en la activación del material; ayuda a la modificación química de la superficie del carbón ayudando a su oxidación, incrementando más grupos superficiales oxigenados produciendo una estructura hidrofílica. (Rodríguez, Giraldo, y Moreno, 2014)

El objetivo central fue establecer una metodología rápida y efectiva, en el proceso de activación del carbón mineral, para ser usado como adsorbente de contaminantes.

\section{MATERIALES Y MÉTODOS}

El presente trabajo se realizó como prueba experimental a nivel de laboratorio, usando los laboratorios de la escuela de Química de la UNMSM y solicitando los servicios del laboratorio químico LABICER de la UNI.

\subsection{Materiales}

Para la activación se necesitó hacer uso de Peróxido de Hidrógeno de J.T. Baker al 30\%, baño ultrasón- ico Branson 1510, horno microondas doméstico marca Samsung de 1200w de potencia.

\subsection{Trituración del carbón mineral}

Se realizó la trituración de $120 \mathrm{gr}$, de carbón mineral, donde se utilizó un mortero y pilón cerámico, para luego ser almacenadas en una bolsa con cierre tipo ziploc; no se midió el tamaño de partícula, por lo que se intentó triturar al tamaño mínimo posible.

\subsection{Lavado de carbón triturado}

Durante la etapa de eliminación de las impurezas existentes en el carbón, entre ellas minerales de pirita o sulfuro de hierro, se requirió hacer uso de un baño ultrasónico y de ácido clorhídrico al $10 \%$. (Xuemin et al., 2015)

El carbono triturado, fue sumergido en el ácido clorhídrico para que dicha mezcla sea sometida a ultrasonido por un tiempo determinado, el ácido y el sonicado ayudan a que el sulfuro férrico se desprenda de la estructura del carbón mineral, pero al mismo tiempo acidifican al material, por lo que al terminar de sonicar el material, se procedió a enjuagar con agua destilada; esta actividad ayuda a retirar las porciones del ácido, así mismo ayuda a regular el $\mathrm{pH}$ del material $(\mathrm{pH}=7)$, una vez el $\mathrm{pH}$ regulado, el material se seca por 24 horas a $60^{\circ} \mathrm{C}$, esta actividad se realizó en el Laboratorio de Química PUCP, por la Lic. Jenny Álvarez, coautora de este artículo.

\subsection{Activación del carbón mineral}

Se pesó $1 \mathrm{gr}$ de material, el cual fue sumergido en $100 \mathrm{ml}$ de peróxido de hidrógeno al 30\%, luego fue llevado al baño ultrasónico para homogenizar la mezcla por un periodo de 5 minutos; transcurrido este tiempo, la muestra fue activada por radiación de microondas por dos minutos con pausas de un minuto, para evitar aumentos excesivos de temperatura; se hizo uso de un horno microondas casero con una potencia de $1200 \mathrm{~W}$, pasado dicho tiempo, se eliminó el peróxido y la muestra se secó a $60^{\circ} \mathrm{C}$ por 24 horas (De la Luz, Sánchez, Martínez, Castaño, y Velazco, 2015)

\subsection{Cinéticas de remoción}

Se trabajó con dos escenarios para generar la comparación de remoción del material, esta se realizó comparando la remoción de cromo total con carbón mineral sin activar, y la segunda con carbón mineral activada. 
Las lecturas se realizaron con el método de absorción atómica, realizando las lecturas cada $20 \mathrm{mi}-$ nutos, durante 3 horas y 20 minutos de duración, actividad realizada en el Laboratorio químico LABICER - UNI.

Las pruebas se realizaron bajo los siguientes parámetros:

- Velocidad de agitación: 500 rpm

- Volumen de solución de cromo (Cr): $50 \mathrm{ml}$

- Concentración de solución de cromo (Cr): 40 ppm

- $\mathrm{pH}$ de solución de cromo: 2.50

- Temperatura: $20.9^{\circ} \mathrm{C}$

- Peso de carbón sin activar: $0.25007 \mathrm{~g}$

- Peso de carbón activado: $0.25012 \mathrm{~g}$

- Método de determinación de cromo total: APHA $3111 \mathrm{~B}$

\section{RESULTADOS Y DISCUSIONES}

En la Figura 1, se muestra la cinética de remoción de cromo total, obtenidas para el carbón sin activar, este llega a un máximo de remoción del $34 \%$ en 200 minutos, empezando a mostrar la saturación del material al minuto 100, donde la curva empieza a ser una constante lineal.

El comportamiento desde el inicio de la prueba es el de remover bajos porcentajes, a comparación del carbón activado (Ver Figura 2), esto no desmerece la capacidad de adsorbente natural que posee el carbón.

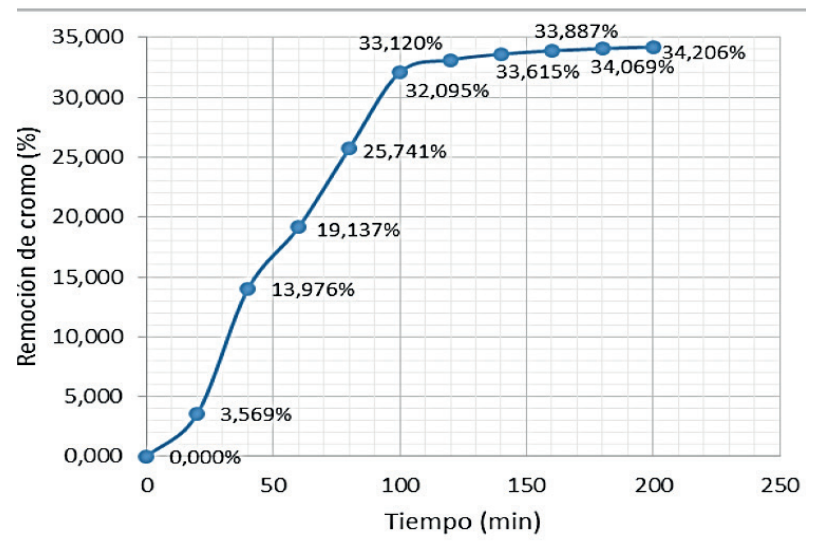

Figura 1. Remoción de Cromo total 40ppm con Carbón sin activar

Fuente: Elaboración propia (2015)

La Figura 2 muestra la curva de remoción de cromo, obtenidas para el carbón activado, este material exhibe un comportamiento conveniente en la remoción del contaminante, presentando un porcentaje mayor desde el inicio de la cinética, a comparación del carbón sin activar (Ver Figura 1); esto debido al incremento de cadenas oxigenadas por la oxidación generada con el peróxido de hidrogeno y el mejoramiento de los poros; producida por la radiación del microondas, condiciones que favorecieron a la activación del carbón.

Esta experiencia hace que el material activado remueva hasta un $41 \%$ en el minuto 200 , tiempo en que se verifica que llega a su capacidad máxima de remoción, por lo que la saturación del material se exhibe en el comportamiento de la curva, empezado a ser lineal.

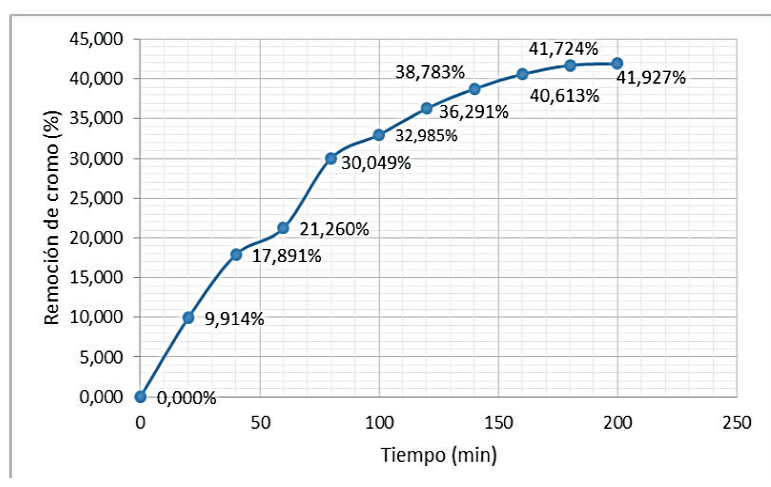

Figura 2. Remoción de Cr Total 40ppm con Carbón Activado Fuente: Elaboración propia (2015)

\section{CONCLUSIONES}

Considerar tiempos más prolongados de activación por microondas, sería un parámetro importante, ya que en la literatura, se detalla que se llegó a un máximo de 2 horas de activación.

Realizar cinéticas de remoción de contaminantes en efluentes reales y donde exista competencia de iones, dará resultados más reales sobre la utilidad del carbón mineral, activado por microondas.

\section{RECOMENDACIONES}

Es recomendable continuar la investigación de este material, con la activación propuesta, modificando los parámetros de análisis y remoción de otro tipo de contaminantes.

Se hace necesario realizar análisis de caracterización del carbón en las diferentes etapas del proceso para su activación, mediante radiación de microondas; considerando su origen mineral.

\section{REFERENCIAS BIBLIOGRÁFICAS}

[1] Agramonte, J., \& Díaz, A. (1983). Inventario Preliminar del Carbón Mineral en el Perú. Lima, Lima, Perú: Instituto Geológico Minero y Metalurgico del Perú. 
[2] Aramburú, V., Núñez, P., Azañero, Á., León, E., Fernández, S., Gagliuffi, P., y otros. (2007). Disminución del contenido de azufre en carbón mineral, mediante procesos metalúrgicos para uso industrial. Revista del Instituto de Investigaciones FIGMMG, 10(20), 8.

[3] Agency for Toxic Substances and Disease Registry (2008). ToxFAQs sobre el cromo. Atlanta, Estados Unidos.

[4] Carbotecnia. (2016). Carbotecnia. Obtenido de http://www.carbotecnia.info/encyclopedia/ que-es-el-carbon-activado/

[5] De la Luz, M., Sánchez, V., Martínez, A., Castaño, V., \& Velazco, C. (2015). Adsorption of phenol from aqueous solutions by carbon nanomaterials of one and two dimensions: Kinetic and Equilibrium studies. Journal of Nanomaterials, 2015, 3-4.

[6] Palacios, A., \& América, L. (1997). Introducción a la toxicología ambiental. En OPS/OMS (Ed.), Introducción a la toxicología ambiental (págs. 227-46). Metepec, México.
[7] Rodríguez, P., Giraldo, L., \& Moreno, J. C. (2014). Modificación de la química superficial de carbones activados. Efecto de la oxidación con soluciones de HNO3 y H2O2 sobre la remoción de Cadmio (II) en solución acuosa. AFINIDAD LXXI, 565, 50-51.

[8] Universidad de Sevilla. (2009). Manual del Carbón Activo. Sevilla, España.

[9] Vaishakh, N., \& Vinu, R. (2016). Peroxide-assisted microwave activation of pyrolysis char for adsorption of dyes from wastewater. Bioresource Technology, 216, 512.

[10] Xuemin, X., Dandan, L., Yujun, Y., Zhilin, W., Zhansheng, W., \& Giancarlo, C. (2015). Preparation of activated carbon mineral from Xinjiang region coal by microwave activation an its application in naphthalene, phenanthrene, and pyrene adsorption. Journal of the Taiwan Institute of Chemical Engineers, 53, 161. 